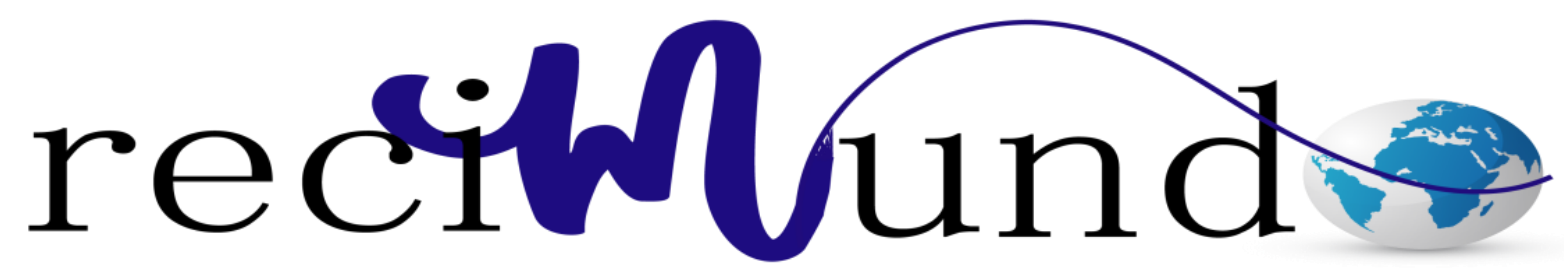

Revista Científica Mundo de la Investigación y el Conocimiento

\author{
Olga Mariela Herrera Checa ${ }^{a}$; Melina Dennise Medina Gamarra ${ }^{\text {b }}$; Marcela \\ Elizabeth Cevallos Sánchez ${ }^{\text {c }}$; María Fernanda Sánchez Pilozo ${ }^{\mathrm{d}}$
}

Derrame pleural de origen tuberculoso

Pleural spill of tuberculose origin

Revista Científica Mundo de la Investigación y el Conocimiento. Vol. 3 núm.3, septiembre, ISSN: 2588-073X, 2019, pp. 275-296

DOI: $\underline{10.26820 / \text { recimundo/3.(3).septiembre.2019.275-296 }}$

URL: http://recimundo.com/index.php/es/article/view/521

Código UNESCO: 3205 Medicina Interna

Tipo de Investigación: Artículo de Revisión

Editorial Saberes del Conocimiento

Recibido: 15/05/2019

Aceptado: $23 / 06 / 2019$

Publicado: 30/09/2019

Correspondencia: olgamariela_777@ hotmail.com

a. Médico; Investigador Independiente; Guayaquil, Ecuador; olgamariela_777@ hotmail.com

b. Médico; Investigador Independiente; Guayaquil, Ecuador; mely-0109@ hotmail.com

c. Médica Cirujana; Investigador Independiente; Guayaquil, Ecuador; eliza250313@ hotmail.com

d. Médico; Investigador Independiente; Guayaquil, Ecuador; mafercitakris@ gmail.com 


\section{Derrame pleural de origen tuberculoso}

Vol. 3, núm. 3., (2019)

Olga Mariela Herrera Checa; Melina Dennise Medina Gamarra; Marcela Elizabeth Cevallos Sánchez; María Fernanda Sánchez Pilozo

\section{RESUMEN}

A escala mundial, la tuberculosis sigue siendo una de las causas más frecuentes de derrames pleurales. La comprensión de la patogénesis de la enfermedad ha evolucionado y ahora se cree que lo que alguna vez se pensó que era un derrame como resultado de una reacción de hipersensibilidad retardada pura, es ahora la consecuencia de la infección directa del espacio pleural con una serie de eventos que incluyen una respuesta inmunológica. La afectación pulmonar es más común de lo que se creía anteriormente y el esputo inducido, puede ser diagnóstico en aproximadamente el 50\% de las personas que la padecen. El estándar de oro para el diagnóstico de pleuritis tuberculosa sigue siendo la detección de Mycobacterium tuberculosis en líquido pleural, o muestras de biopsia pleural, ya sea por microscopía y / o cultivo, o la demostración histológica de granulomas caseificantes en la pleura junto con bacilos ácidos rápidos. Sin embargo, en entornos de alta carga, el diagnóstico se infiere con frecuencia en pacientes que presentan un exudado predominantemente linfocítico y un nivel alto de adenosina desaminasa (ADA), que es un complemento valioso en la evaluación diagnóstica. La ADA generalmente es fácilmente accesible y, junto con el predominio de linfocitos, justifica el inicio del tratamiento en pacientes con una alta probabilidad de pre-test. Aun así, los resultados falsos negativos y falsos positivos siguen siendo un problema. Cuando se agrega una biopsia pleural cerrada al recuento de ADA y linfocitos, la precisión diagnóstica se aproxima a la de la toracoscopia. El papel de otros biomarcadores está menos descrito. El drenaje pleural temprano puede tener un papel en casos seleccionados, pero se requiere más investigación para validar su uso y definir la subpoblación que puede beneficiarse de tales intervenciones.

Palabras clave: Derrame pleural; Tuberculosis (TB); Toracocentesis; Biopsia pleural. 


\title{
Derrame pleural de origen tuberculoso
}

Vol. 3, núm. 3., (2019)

Olga Mariela Herrera Checa; Melina Dennise Medina Gamarra; Marcela Elizabeth Cevallos Sánchez; María Fernanda Sánchez Pilozo

\begin{abstract}
Globally, tuberculosis remains one of the most frequent causes of pleural effusions. The understanding of the pathogenesis of the disease has evolved and it is now believed that what has ever been determined to be a stroke as a result of a reaction of pure delayed hypersensitivity, is now the consequence of direct infection of the pleural space with a series of events that include an immune response. Pulmonary involvement is more common than previously believed and induced sputum can be diagnostic in approximately $50 \%$ of people who suffer. The gold standard for the diagnosis of tuberculous pleurisy remains the detection of Mycobacterium tuberculosis in pleural fluid, or samples of pleural biopsy, either by microscopy and / or culture, or the histological demonstration of caseifying granulomas in the pleura together with bacillus symptoms rapid. However, in high-load settings, the diagnosis is frequently inferred in patients who present with predominantly lymphocytic exudate and a high level of adenosine deaminase (ADA), which is a valuable complement in the diagnostic evaluation. The ADA is usually easily accessible and, together with the predominance of lymphocytes, justifies the start of treatment in patients with a high probability of pretest. Still, false negative and false positive results remain a problem. When a closed pleural biopsy is added to the ADA and lymphocyte count, the diagnostic accuracy approximates that of thoracoscopy. The role of other biomarkers is less described. Early pleural drainage may have a role in selected cases, but more research is required to validate its use and define the subpopulation that can benefit stories.
\end{abstract}

Key words: Pleural effusion; Tuberculosis (TB); Thoracentesis; Pleural biopsy. 


\section{Derrame pleural de origen tuberculoso}

Vol. 3, núm. 3., (2019)

Olga Mariela Herrera Checa; Melina Dennise Medina Gamarra; Marcela Elizabeth Cevallos Sánchez; María Fernanda Sánchez Pilozo

\section{Introducción.}

La tuberculosis (TB) ha sido tradicionalmente una de las principales causas de enfermedad pleural y hasta las primeras décadas del siglo pasado se mantuvieron como un paradigma principal de la "pleuresía". De hecho, en la presencia de un derrame claramente exudativo y una presentación clínica compatible el término utilizado "pleuritis exudativa" insinuó una etiología tuberculosa y, por lo tanto, ha sido entendido como sinónimo de "pleuritis exudativa tuberculosa".

El autor (Dolin \& Raviglione, 2010) expresa que en la era de la tuberculosis mencionar el término "pleuresía exudativa" era de gran medida, sin embargo, hoy en día se debe describir los derrames exudativos en general, por lo tanto, se sugiere el término preciso "pleuresía exudativa tuberculosa" siempre que sea deba a tuberculosis. De lo contrario, el término "pleuresía tuberculosa" o "Pleuritis tuberculosa" se utiliza para describir esta entidad, en algunos países también el término "específico pleuresía "es común.

Además de la pleuritis aguda tuberculosa exudativa, la tuberculosis de la pleura puede sin embargo, presentarse como una enfermedad bastante crónica en términos de pleuresía caseosa o específica (es decir, tuberculoso) respectivamente.

Los derrames pleurales de acuerdo al autor (Richard, 2017) se definen como acumulaciones de líquido dentro del espacio pleural. Tienen múltiples causas y en general se clasifican como trasudados o exudados. La detección es por examen físico y radiografía de tórax; para determinar la causa, a menudo se requieren la toracocentesis y el análisis del líquido pleural. Los trasudados 


\section{Derrame pleural de origen tuberculoso}

Vol. 3, núm. 3., (2019)

Olga Mariela Herrera Checa; Melina Dennise Medina Gamarra; Marcela Elizabeth Cevallos Sánchez; María Fernanda Sánchez Pilozo

asintomáticos no precisan tratamiento. Los sintomáticos y casi todos los exudados requieren toracocentesis, drenaje con tubo de tórax, pleurodesis o pleurectomía.

La tuberculosis es una bacteria patógena conocida como Mycobacterium tuberculosis que pertenece a la familia Mycobacteriaceae. A pesar de estar aislada por Robert Koch en 1882, la tuberculosis sigue siendo una de las enfermedades transmisibles más mortales. (Smith, 2003)

En 2013, se estima que 9 millones de personas desarrollaron TB activa, con 1,5 millones de muertes atribuidas a la enfermedad. Según la Organización Mundial de la Salud, la incidencia de TB pulmonar en algunas regiones es tan alta como 1,000 casos por cada 100,000 personas. (OMS, 2014)

Aunque la tuberculosis afecta los pulmones en la mayoría de los pacientes, la tuberculosis extrapulmonar sirve como presentación inicial en aproximadamente el $25 \%$ de los adultos y afecta principalmente a los ganglios linfáticos y la pleura.

Esta revisión ofrece una visión general de la patogénesis, presentación clínica, diagnóstico y tratamiento de los derrames pleurales de TB, destacando los avances y controversias reciente.

\section{Métodos y materiales.}

Para el desarrollo de este proceso investigativo, se plantea como metodología la encaminada hacia una orientación científica particular que se encuentra determinada por la necesidad de indagar en forma precisa y coherente una situación, en tal sentido (Davila, 2015) 


\section{Derrame pleural de origen tuberculoso}

Vol. 3, núm. 3., (2019)

Olga Mariela Herrera Checa; Melina Dennise Medina Gamarra; Marcela Elizabeth Cevallos Sánchez; María Fernanda Sánchez Pilozo

define la metodología "como aquellos pasos previos que son seleccionados por el investigador para lograr resultados favorables que le ayuden a plantear nuevas ideas”. (p.66)

Lo citado por el autor, lleva a entender que el desarrollo de la acción investigativa busca simplemente coordinar acciones enmarcadas en una revisión bibliográfica con el fin de complementar ideas previas relacionadas Derrame pleural de origen tuberculoso, a través, de una revisión de literatura, para así finalmente elaborar un cuerpo de consideraciones generales que ayuden a ampliar el interés propuesto.

\section{Tipo de Investigación.}

Dentro de toda práctica investigativa, se precisan acciones de carácter metodológico mediante las cuales, se logra conocer y proyectar los eventos posibles que la determinan, así como las características que hacen del acto científico un proceso interactivo ajustado a una realidad posible de ser interpretada. En este sentido, se puede decir, que la presente investigación corresponde al tipo documental, definido por Castro (2016), "se ocupa del estudio de problemas planteados a nivel teórico, la información requerida para abordarlos se encuentra básicamente en materiales impresos, audiovisuales y /o electrónicos”. (p.41).

En consideración a esta definición, la orientación metodológica permitió la oportunidad de cumplir con una serie de actividades inherentes a la revisión y lectura de diversos documentos donde se encontraron ideas explicitas relacionadas con los tópicos encargados de identificar a cada característica insertada en el estudio. Por lo tanto, se realizaron continuas interpretaciones con el claro propósito de revisar aquellas apreciaciones o investigaciones propuestas por diferentes 


\section{Derrame pleural de origen tuberculoso}

Vol. 3, núm. 3., (2019)

Olga Mariela Herrera Checa; Melina Dennise Medina Gamarra; Marcela Elizabeth Cevallos Sánchez; María Fernanda Sánchez Pilozo

investigadores relacionadas con el tema de interés, para luego dar la respectiva argumentación a los planteamientos, en función a las necesidades encontradas en la indagación.

\section{Fuentes documentales}

El análisis correspondiente a las características que predomina en el tema seleccionado, llevan a incluir diferentes fuentes documentales encargadas de darle el respectivo apoyo y en ese sentido cumplir con la valoración de los hechos a fin de generar nuevos criterios que sirven de referencia a otros procesos investigativos. Para (CASTRO, 2016) las fuentes documentales incorporadas en la investigación documental o bibliográfica, "representa la suma de materiales sistemáticos que son revisados en forma rigurosa y profunda para llegar a un análisis del fenómeno".(p.41). Por lo tanto, se procedió a cumplir con la realización de una lectura previa determinada para encontrar aquellos aspectos estrechamente vinculados con el tema, con el fin de explicar mediante un desarrollo las respectivas apreciaciones generales de importancia.

\section{Técnicas para la Recolección de la Información}

La conducción de la investigación para ser realizada en función a las particularidades que determinan a los estudios documentales, tiene como fin el desarrollo de un conjunto de acciones encargadas de llevar a la selección de técnicas estrechamente vinculadas con las características del estudio. En tal sentido, (Bolívar, 2015), refiere, que es "una técnica particular para aportar ayuda a los procedimientos de selección de las ideas primarias y secundarias”. (p. 71).

Por ello, se procedió a la utilización del subrayado, resúmenes, fichaje, como parte básica para la revisión y selección de los documentos que presentan el contenido teórico. Es decir, que 


\section{Derrame pleural de origen tuberculoso}

Vol. 3, núm. 3., (2019)

Olga Mariela Herrera Checa; Melina Dennise Medina Gamarra; Marcela Elizabeth Cevallos Sánchez; María Fernanda Sánchez Pilozo

mediante la aplicación de estas técnicas se pudo llegar a recoger informaciones en cuanto a la revisión bibliográfica de los diversos elementos encargados de orientar el proceso de investigación. Tal como lo expresa, (Bolívar, 2015) "las técnicas documentales proporcionan las herramientas esenciales y determinantes para responder a los objetivos formulados y llegar a resultados efectivos" (p. 58). Es decir, para responder con eficiencia a las necesidades investigativas, se introdujeron como técnica de recolección el método inductivo, que hizo posible llevar a cabo una valoración de los hechos de forma particular para llegar a la explicación desde una visión general.

Asimismo, se emplearon las técnicas de análisis de información para la realización de la investigación que fue ejecutada bajo la dinámica de aplicar diversos elementos encargados de determinar el camino a recorrer por el estudio, según, (Bolívar, 2015) las técnicas de procesamiento de datos en los estudios documentales "son las encargadas de ofrecer al investigador la visión o pasos que debe cumplir durante su ejercicio, cada una de ellas debe estar en correspondencia con el nivel a emplear" (p. 123). Esto indica, que para llevar a cabo el procesamiento de los datos obtenidos una vez aplicado las técnicas seleccionadas, tales como: fichas de resumen, textual, registros descriptivos entre otros, los mismos se deben ajustar al nivel que ha sido seleccionado.

\section{Resultados.}

Patogénesis 


\section{Derrame pleural de origen tuberculoso}

Vol. 3, núm. 3., (2019)

Olga Mariela Herrera Checa; Melina Dennise Medina Gamarra; Marcela Elizabeth Cevallos Sánchez; María Fernanda Sánchez Pilozo

Hasta hace poco, se pensaba que los derrames pleurales de TB se producían en gran parte como resultado de una reacción de hipersensibilidad retardada. La inyección de tuberculina en la cavidad pleural de los cobayas sensibilizados con M. tuberculosis, produce un gran derrame pleural rico en proteínas durante un período de 24 horas, que es completamente suprimido por el suero de antilinfocitos. (Leibowitz, Kennedy, \& Lessof, 2013). Según este modelo y el hecho de que los investigadores no pudieron cultivar M. tuberculosis a partir de fluidos pleurales, se presumió que la patogénesis se debía a una hipersensibilidad retardada en lugar de una infección directa del espacio pleural. Con la llegada de medios de cultivo mejorados, ahora es posible cultivar M. tuberculosis tanto del líquido pleural como del tejido pleural en hasta el $70 \%$ de los casos, y siguiendo el postulado de infección de Koch, esto sugeriría una relación causal. (Koch, 1990)

El derrame pleural es probablemente una manifestación de infección micobacteriana paucibacilar dentro del espacio pleural, que se adquiere de las lesiones parenquimatosas iniciales y da como resultado una respuesta inmunológica que aumenta la formación de líquido pleural y disminuye la eliminación del líquido pleural. (Seibert, Haynes, Middleton, \& R, 2012). Inicialmente, hay una respuesta inflamatoria neutrofílica rápida dentro de la pleura que es sintomática. Esto es seguido por una reacción inmune impulsada por linfocitos prolongada que se acompaña de formación de granuloma pleural y liberación de adenosina desaminasa (ADA). Por lo tanto, es plausible que la probabilidad de un cultivo positivo de líquido pleural disminuya con el tiempo, ya que el derrame se vuelve predominante en los linfocitos y se contienen micobacterias viables. 


\section{Derrame pleural de origen tuberculoso}

Vol. 3, núm. 3., (2019)

Olga Mariela Herrera Checa; Melina Dennise Medina Gamarra; Marcela Elizabeth Cevallos Sánchez; María Fernanda Sánchez Pilozo

\section{Manifestaciones clínicas}

La enfermedad pleural es la segunda después de la afectación linfática como sitio de TB extrapulmonar, y puede ocurrir en la enfermedad primaria o en la reactivación. En los EE. UU.,

Se informa que el 3-5\% de los pacientes con TB tienen enfermedad pleural, mientras que la incidencia de afectación pleural puede llegar al 30\% en entornos de TB de alta carga (Baumann, Nolan, Petrini, \& M, 2007).

Cuando el VIH es endémico, la pleuritis tuberculosa es la causa más común de derrame linfocítico y se cree que es causada por una infección primaria en el $30 \%$ de los pacientes. La mayoría de los pacientes (VIH negativos) con derrames pleurales de TB presentarán una enfermedad febril aguda caracterizada por una tos no productiva y dolor torácico pleurítico, pero sin una elevación en el recuento periférico de glóbulos blancos. (Onyenekwu, 2013). También se informa con frecuencia sudoración nocturna, escalofríos, debilidad, disnea y pérdida de peso.

El derrame pleural de TB puede resolverse espontáneamente sin tratamiento, pero los pacientes con frecuencia desarrollan TB activa en una fecha posterior. En áreas endémicas sin VIH donde la reactivación es el mecanismo predominante de la enfermedad de TB, se informa que la afectación pleural ocurre en el 4\% de los casos. Dichos pacientes tienen un inicio más insidioso, son de mayor edad y tienen más probabilidades de estar inmunocomprometidos. Se ha demostrado que los pacientes inmunocomprometidos tienen tasas de cultivo positivas más altas, que se cree que se deben a la limpieza deteriorada del organismo. (Bolliger, 2015) 


\section{Derrame pleural de origen tuberculoso}

Vol. 3, núm. 3., (2019)

Olga Mariela Herrera Checa; Melina Dennise Medina Gamarra; Marcela Elizabeth Cevallos Sánchez; María Fernanda Sánchez Pilozo

El autor (Turna, 2006) en su estudio expresa que el empiema de tuberculosis crónica es menos común y representa una entidad distinta de infección crónica activa en el espacio pleural. Se caracteriza por un líquido purulento donde prácticamente todos los glóbulos blancos nucleados son neutrófilos, y pueden ocurrir en varios entornos:

(I) Progresión de una pleuritis primaria por tuberculosis.

(II) Extensión directa de la infección al espacio pleural desde los ganglios linfáticos torácicos o un foco subdiafragmático

(III) Diseminación hematógena; o

(IV) después de una neumonectomía.

La mayoría de los empiemas se resolverán dejando una pleura engrosada, cicatrizada y calcificada. Sin embargo, este proceso puede complicarse por la descompresión a través de la pared torácica (empiema necesario). El neumotórax secundario a la TB a menudo anuncia una grave afectación pulmonar por el proceso infeccioso y la aparición de fístula broncopleural y empiema. (Turna, 2006)

La fibrosis pleural o el fibrotórax (Figura 1) es una complicación bien descrita de la pleuritis tuberculosa. Sin embargo, la incertidumbre sigue siendo la prevalencia exacta, con informes varían entre 5\% y 55\%. Además, la fibrosis pleural puede tener implicaciones clínicas a largo plazo, y algunos estudios informan la asociación del engrosamiento pleural residual ( $\geq 10$ 


\section{Derrame pleural de origen tuberculoso}

Vol. 3, núm. 3., (2019)

Olga Mariela Herrera Checa; Melina Dennise Medina Gamarra; Marcela Elizabeth Cevallos Sánchez; María Fernanda Sánchez Pilozo

$\mathrm{mm}$ ) con una morbilidad significativa, que incluye dolor torácico crónico, disnea y deterioro de la función pulmonar. (Light, 2010)

Figura $\mathbf{N}^{\mathbf{0}}$ 1. Caso grave de fibrotórax como una complicación a largo plazo de la pleuritis por tuberculosis. TB, tuberculosis.

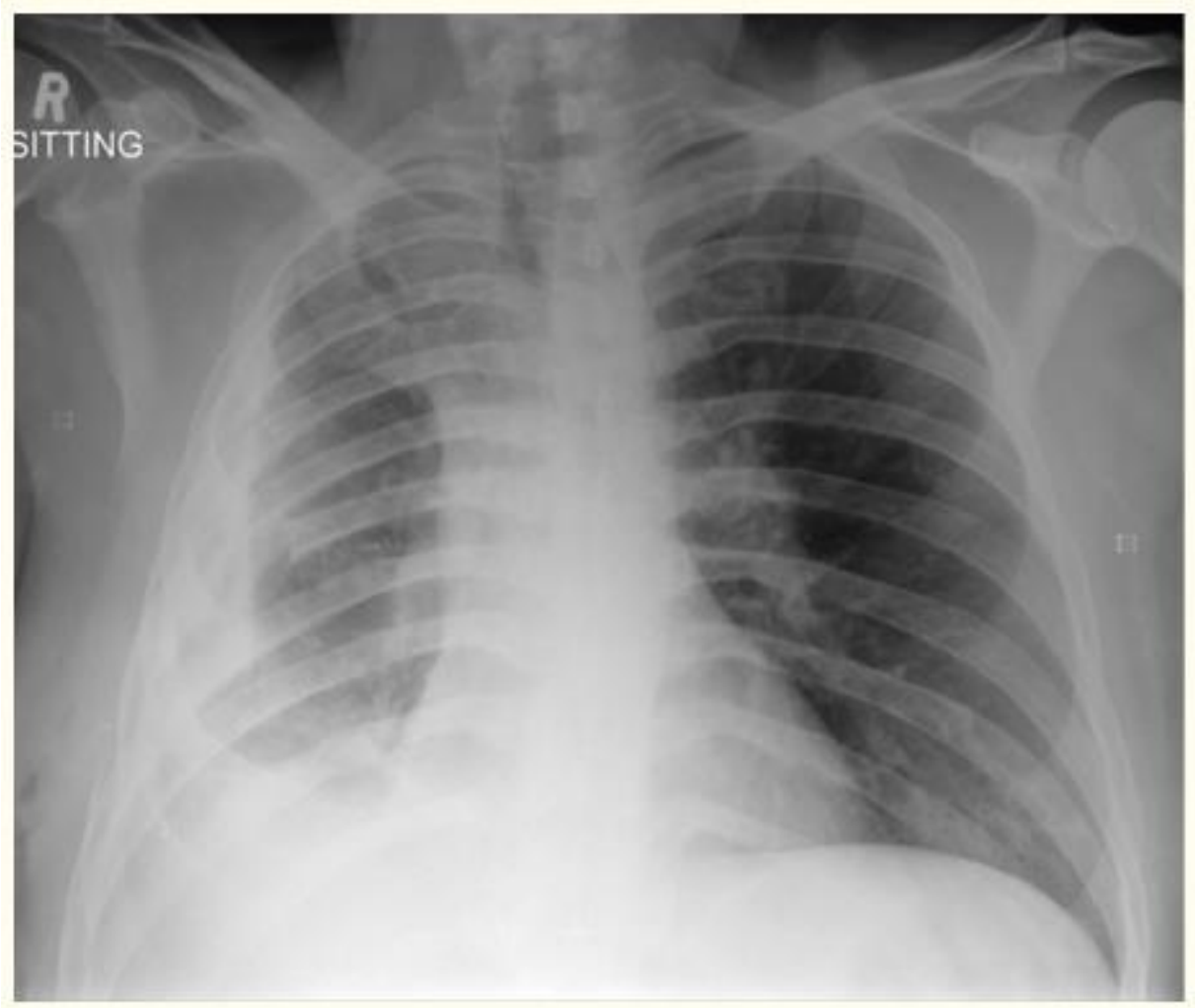

Fuente: (Light, 2010).

$\underline{\text { Radiografía de pecho }}$

Los derrames pleurales secundarios a la tuberculosis son en gran medida unilaterales con un ligero predominio del lado derecho, que se informa que ocurren en el 55\% de los casos (Figura 2). Los derrames son típicamente de tamaño pequeño a moderado, ocupando menos de 


\section{Derrame pleural de origen tuberculoso}

Vol. 3, núm. 3., (2019)

Olga Mariela Herrera Checa; Melina Dennise Medina Gamarra; Marcela Elizabeth Cevallos

Sánchez; María Fernanda Sánchez Pilozo

un tercio del hemitórax en aproximadamente el $80 \%$ de los casos. Sin embargo, ni el tamaño ni el lado del derrame han tenido relación con el pronóstico. (Sibley, 2000)

Figura N². Paciente con tuberculosis pleural confirmada. No se pudieron observar cambios parenquimatosos significativos. TB, tuberculosis.

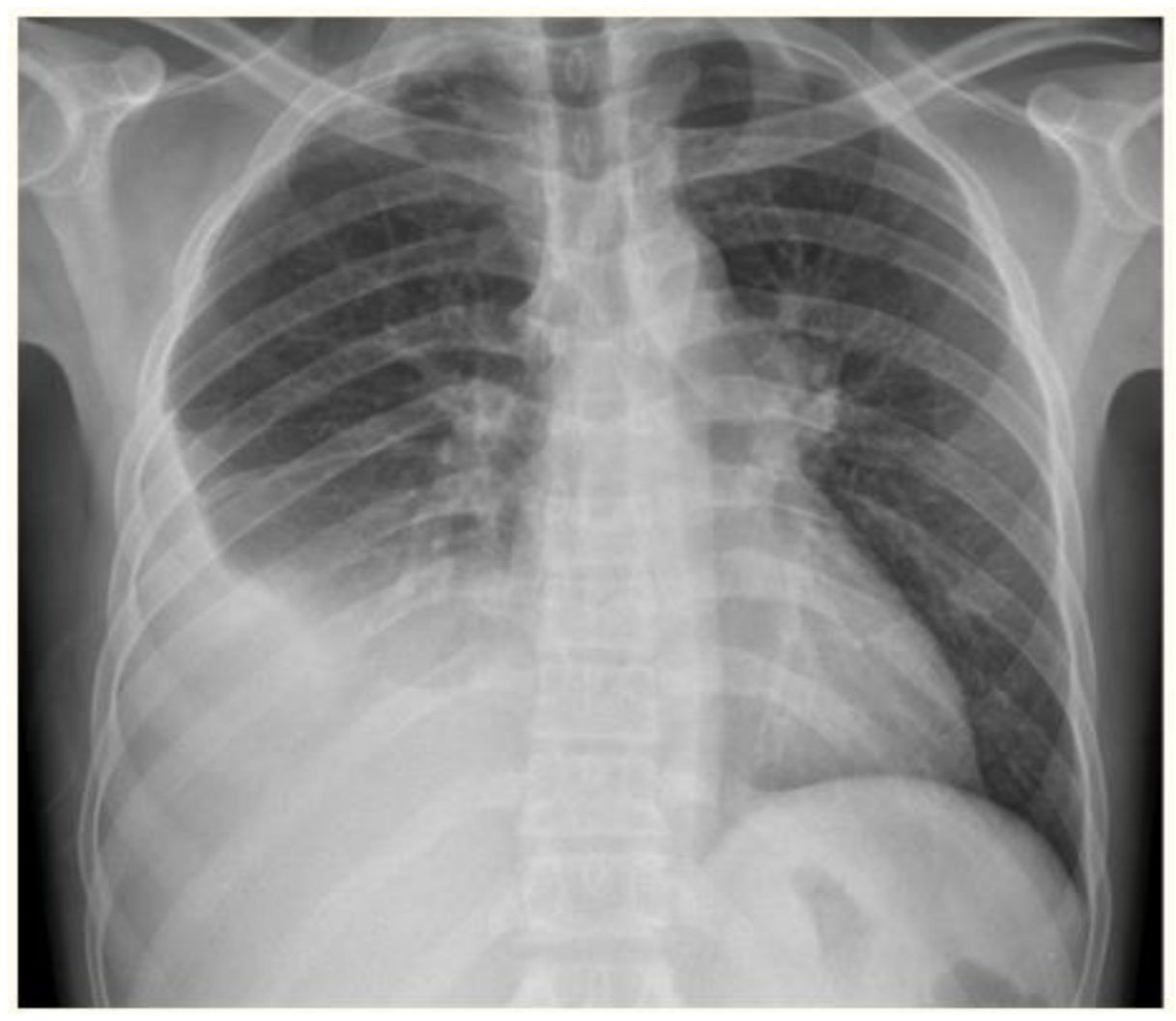

Fuente: (Sibley, 2000).

\section{$\underline{\text { Ultrasonido }}$}

La ecografía torácica ahora se acepta como estándar de atención en la realización de toracocentesis y biopsias pleurales cerradas. La detección del engrosamiento pleural localizado y otras anomalías pleurales puede dirigir al operador a un sitio de biopsia preferido. Sin embargo, 


\section{Derrame pleural de origen tuberculoso}

Vol. 3, núm. 3., (2019)

Olga Mariela Herrera Checa; Melina Dennise Medina Gamarra; Marcela Elizabeth Cevallos Sánchez; María Fernanda Sánchez Pilozo

además de la realización de procedimientos de diagnóstico invasivos, la ecografía también puede ayudar a caracterizar la naturaleza del derrame. La apariencia ecográfica de los derrames pleurales secundarios a la TB varía desde anecoicos a complejos separados o no septados a incluso derrames homogéneamente ecogénicos. (Theron, 2010)

Tomografía computarizada (CT)

La tomografía computarizada del tórax es actualmente la mejor modalidad de imagen para visualizar tanto la pleura como el parénquima pulmonar en los derrames pleurales de la tuberculosis (Figura 3). Además de visualizar el alcance de la enfermedad, la TC se puede utilizar para evaluar el empiema de la tuberculosis, que se puede dividir en tres fases distintas. La fase exudativa representa el derrame inicial sin complicaciones, seguido de la fase fibrinopurulenta, donde la TC generalmente muestra pleuras viscerales y parietales engrosadas separadas por líquido, conocido como el signo de "pleura dividida". En la fase de organización, la TC revela una colección de líquido pleural loculado con una exfoliación pleural engrosada y un grado variable de calcificación con o sin proliferación de grasa extrapleural. (Candela, 2003)

Figura $\mathbf{N}^{\mathbf{0} 3}$. TC de paciente con derrame pleural confirmado de tuberculosis con afectación parenquimatosa (consolidación subsegmental en este caso particular). 


\section{Derrame pleural de origen tuberculoso}

Vol. 3, núm. 3., (2019)

Olga Mariela Herrera Checa; Melina Dennise Medina Gamarra; Marcela Elizabeth Cevallos

Sánchez; María Fernanda Sánchez Pilozo

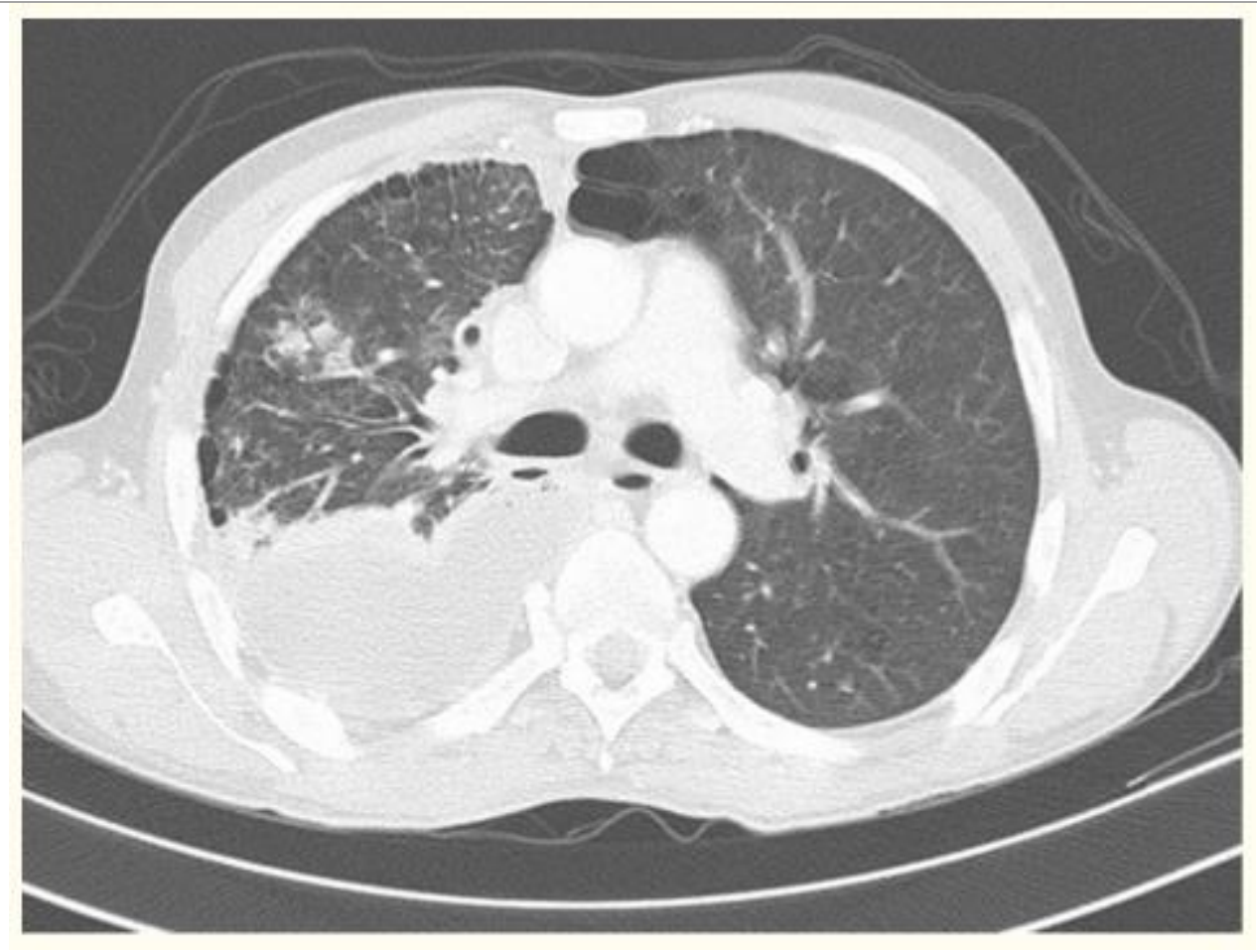

Fuente: (Candela, 2003).

\section{Diagnóstico.}

La prueba más eficaz para diagnóstico de pleuritis tuberculosa es la detección de M. tuberculosis en el esputo, el líquido pleural o las muestras de biopsia pleural, ya sea por microscopía y / o cultivo, o la demostración histológica de granulomas caseificantes en la pleura junto con bacilos ácidos rápidos (AFB) (Light, 2010). Sin embargo, en entornos de alta carga, el diagnóstico se infiere con frecuencia en pacientes que presentan un exudado predominantemente linfocítico y un alto nivel de ADA.

- Esputo 


\section{Derrame pleural de origen tuberculoso}

Vol. 3, núm. 3., (2019)

Olga Mariela Herrera Checa; Melina Dennise Medina Gamarra; Marcela Elizabeth Cevallos Sánchez; María Fernanda Sánchez Pilozo

Anteriormente se creía que los pacientes sin lesión parenquimatosa manifestante coexistente son esputo negativo y, por lo tanto, no contagiosos. Además, la tasa de cultivo de micobacterias en el esputo no inducido es baja. Las sensibilidades reportadas varían de 0\% a 30\% (Ko, Park, \& Kim, 2014). El método de recolección de esputo, sin embargo, es de crucial importancia.

Los autores (Conde \& Loivos, 2003) informaron un rendimiento del $52 \%$ en cultivo de micobacterias con muestras de esputo inducidas. Incluso en pacientes con parénquima pulmonar subyacente normal en la radiografía de tórax, el rendimiento del cultivo de esputo en muestras inducidas se acercó al 55\%. Por lo tanto, en pacientes con sospecha de derrame pleural de TB, es importante obtener cultivos en muestras de esputo inducido, incluso en ausencia de compromiso evidente del parénquima.

- Toracocentesis (Microscopía y cultivo).

La apariencia macroscópica es la del líquido de color pajizo en más del 80\% de los casos. La microscopía para AFB en el líquido pleural puede identificar M. tuberculosis en menos del 10\% de los casos. La excepción a esto son los pacientes con VIH y empiema tuberculoso, donde los rendimientos pueden ser mayores (> 20\%) (Gopi, 2006). El cultivo del líquido pleural se puede realizar en medios sólidos o líquidos como en el sistema semiautomatizado BACTEC MGIT, ampliamente disponible y comercial, o con métodos de cultivo manuales que pueden permitir la resistencia de la prueba al mismo tiempo, como el ensayo de susceptibilidad al fármaco de observación microscópica (MODS) (Tovar, 2008). Cuando se usan medios de cultivo sólidos, las sensibilidades reportadas han sido bajas, en el rango de $12 \%$ a $30 \%$. Sin embargo, parece que los 


\section{Derrame pleural de origen tuberculoso}

Vol. 3, núm. 3., (2019)

Olga Mariela Herrera Checa; Melina Dennise Medina Gamarra; Marcela Elizabeth Cevallos Sánchez; María Fernanda Sánchez Pilozo

medios de cultivo líquidos muestran mejores sensibilidades de hasta el $70 \%$. Un beneficio adicional del uso de medios líquidos es el tiempo significativamente más corto requerido para los resultados del cultivo, que es de 2 semanas en comparación con las 6 semanas tradicionales para medios sólidos

- Biopsia pleural.

La presencia de granulomas caseificantes que contienen bacilos ácido-rápidos en el examen histológico de la superficie pleural es diagnóstico de pleuritis tuberculosa (Gopi, 2006). La demostración de bacilos acidorresistentes no es un requisito absoluto; la presencia de granulomas caseificantes en entornos de alta carga se considera adecuada.

El tejido pleural se puede cosechar mediante biopsias pleurales cerradas, toracoscopia o biopsias quirúrgicas abiertas (Bolliger, 2015). El acceso a la toracoscopia y las biopsias quirúrgicas abiertas es limitado en muchas partes del mundo donde la TB es endémica y, por lo tanto, si el análisis del líquido pleural no es concluyente, la biopsia cerrada es la próxima investigación preferida. Las técnicas de biopsia guiadas por ultrasonido han ganado popularidad entre los médicos pulmonares y se están convirtiendo en el estándar de atención para obtener tejido pleural en pacientes con sospecha de pleuritis tuberculosa.

La toracoscopia médica se reserva para el pequeño número de casos en los que las biopsias pleurales cerradas no pueden proporcionar un diagnóstico. La biopsia pleural guiada por ultrasonido tiene un rendimiento diagnóstico de hasta $90 \%$ para la TB pleural, lo cual no es sorprendente dada la naturaleza difusa de la enfermedad. (Bolliger, 2015) 


\section{Derrame pleural de origen tuberculoso}

Vol. 3, núm. 3., (2019)

Olga Mariela Herrera Checa; Melina Dennise Medina Gamarra; Marcela Elizabeth Cevallos Sánchez; María Fernanda Sánchez Pilozo

Debido al error de muestreo, la sensibilidad generalmente aumenta con el número de biopsias tomadas, y previamente se ha descubierto que las biopsias tomadas durante la toracoscopia médica tienen una sensibilidad diagnóstica del 100\%. Con la toracoscopia médica es posible la visualización directa de la pleura enferma, utilizando un endoscopio rígido o flexible, que guía al operador al sitio de biopsia más apropiado. Debido al alto rendimiento reportado y la naturaleza menos invasiva de la toracoscopia, rara vez se requieren biopsias quirúrgicas abiertas. (Bolliger, 2015)

\section{Tratamiento}

El tratamiento médico para el derrame pleural de TB es el mismo que para la TB pulmonar, y es consistente con la teoría de que la mayoría de los casos de TB pleural se desarrollan a partir de una enfermedad pulmonar. La resolución esperada del derrame pleural de TB es variable, y suponiendo una terapia adecuada, la fiebre generalmente se resuelve en 2 semanas con la reabsorción del líquido pleural en 6 semanas. Naturalmente, esto dependerá de la carga de la enfermedad en el individuo, y el tamaño del derrame y la reabsorción puede demorar hasta 2-4 meses.

La evidencia actual sobre el papel de la intervención quirúrgica es limitada y difícil de interpretar, y el drenaje intercostal tradicionalmente no se ofrece a los pacientes a menos que exista disnea severa (Bagheri, 2013).

Un estudio reciente realizado por (Bhuniya, 2012) investigaron el uso del drenaje pleural temprano (mediante manometría pleural) además de la terapia estándar contra la tuberculosis, en 


\section{Derrame pleural de origen tuberculoso}

Vol. 3, núm. 3., (2019)

Olga Mariela Herrera Checa; Melina Dennise Medina Gamarra; Marcela Elizabeth Cevallos Sánchez; María Fernanda Sánchez Pilozo

comparación con la terapia estándar sola; donde demostraron las diferencias significativas después de 6 meses en la función pulmonar. El grupo de drenaje tuvo un volumen espiratorio forzado en el primer segundo (VEF1) del 87,6\% en comparación con el grupo de control del 84,9\% $(\mathrm{P}=0,02)$, con capacidad vital forzada (CVF) del 84,5\% y 83,3\% (P <0,01), respectivamente. Se desconoce la relevancia clínica a largo plazo de estas pequeñas diferencias en la función pulmonar. Sin embargo, estos autores informaron una menor incidencia de engrosamiento pleural residual en pacientes con drenaje y también comentaron que los pacientes con toracocentesis terapéutica experimentaron un alivio inmediato de la disnea después del drenaje.

Estudios anteriores informaron que el engrosamiento pleural residual $\geq 10 \mathrm{~mm}$ puede causar síntomas clínicos significativos en pacientes con derrame pleural de TB, con incidencias reportadas que varían de $26 \%$ a $50.4 \%$ (Candela, 2003). En datos no publicados, encontramos que los pacientes con derrames pleurales confirmados de $\mathrm{TB}$, aleatorizados para drenaje pleural terapéutico, mostraron mejoras significativamente superiores en varios parámetros de la función pulmonar después de 3 y 6 meses de seguimiento, a pesar de que se logró un drenaje completo en menos de la mitad de todos los pacientes.

En pacientes seleccionados, la administración de corticosteroides puede acortar la duración de la fiebre y el tiempo de reabsorción de líquidos, aunque los riesgos y beneficios de los corticosteroides en este contexto no han sido bien definidos. Actualmente, los datos son insuficientes para respaldar el uso complementario de rutina de los corticosteroides para la pleuritis tuberculosa. (Engel, 2007)

\section{Conclusiones.}




\section{Derrame pleural de origen tuberculoso}

Vol. 3, núm. 3., (2019)

Olga Mariela Herrera Checa; Melina Dennise Medina Gamarra; Marcela Elizabeth Cevallos Sánchez; María Fernanda Sánchez Pilozo

Durante el desarrollo del proceso investigativo se observa que el derrame pleural es una acumulación excesiva de líquido en el espacio pleural. Sin embargo, esto puede presentar un dilema de diagnóstico para el médico tratante porque puede estar relacionado con trastornos del pulmón o la pleura, o con un trastorno sistémico.

También se observó que las personas que padecen esta patología presentan con mayor frecuencia disnea, inicialmente con esfuerzo, tos predominantemente seca y dolor torácico pleurítico. Por esta manera para tratar el derrame pleural de manera adecuado, es importante determinar su etiología.

No obstante, la etiología del derrame pleural sigue sin estar clara en casi el $20 \%$ de los casos, por lo que la toracocentesis se debe realizar para derrames pleurales nuevos e inexplicables y las pruebas de laboratorio ayudan a distinguir el transudado del líquido pleural de un exudado.

La evaluación diagnóstica del derrame pleural de origen tuberculoso determinó que incluye estudios químicos y microbiológicos, así como análisis citológicos que proporcionan mayor información sobre la etiología del proceso de la enfermedad. La inmunohistoquímica proporciona una buena precisión diagnóstica. Los derrames transudativos generalmente se manejan tratando el trastorno médico subyacente. Sin embargo, un derrame pleural refractario grande, ya sea transudado o exudado, debe drenarse para proporcionar alivio sintomático.

El manejo del derrame exudativo depende de la etiología subyacente del derrame. Los derrames malignos generalmente se drenan para paliar los síntomas y pueden requerir pleurodesis para prevenir la recurrencia. La biopsia pleural se recomienda para la evaluación y exclusión de 


\section{Derrame pleural de origen tuberculoso}

Vol. 3, núm. 3., (2019)

Olga Mariela Herrera Checa; Melina Dennise Medina Gamarra; Marcela Elizabeth Cevallos Sánchez; María Fernanda Sánchez Pilozo

diversas etiologías. La biopsia pleural cerrada percutánea es la más fácil de realizar menos costosa, y con complicaciones mínimas, y debe usarse de manera rutinaria.

\section{Referencias Bibliográficas}

Bagheri, R. (2013). Outcomes following surgery for complicated tuberculosis: analysis of 108 patients. Thorac Cardiovasc Surg, 61(2), 54-8.

Baumann, M., Nolan, R., Petrini, \& M. (2007). Pleural tuberculosis in the United States: incidence and drug resistance. Chest, 131(4), 25-32.

Bhuniya, S. (2012). Role of therapeutic thoracentesis in tuberculous pleural effusion. Ann Thorac Med, 7(4), 15-9.

Bolívar, J. (2015). Investigación Documental. México: Pax.

Bolliger, C. (2015). El rendimiento de diferentes volúmenes de líquido pleural para el cultivo de Mycobacterium tuberculosis. Torax.

Candela, A. (2003). Secuelas funcionales de pleuresía tuberculosa en pacientes tratados correctamente. 30-56.

Castro, J. (2016). Técnicas Documentales. México. Limusa.

Conde, M., \& Loivos, A. (2003). Yield of sputum induction in the diagnosis of pleural tuberculosis. . Am J Respir Crit Care Med, 23-5. .

Davila, A. (2015). Diccionario de Términos Científicos. . Caracas: Editorial Oasis.

Dolin, P., \& Raviglione, M. (2010). Global tuberculosis incidence and mortality during. Bull World Health Organ, 72(2), 213-220.

Engel, M. (2007). Corticosteroids for tuberculous pleurisy. Cochrane Database Syst Rev, 14(3).

Gopi, A. (2006). Diagnóstico y tratamiento del derrame pleural tuberculoso en 2006. 80-9.

Ko, J., Park, H., \& Kim, C. (2014). Pulmonary changes of pleural TB: up-to-date CT imaging. 411. .

Koch, R. (1990). Die Aetiologie der Tuberculose. Berliner Klinische Wochenschrift.

Leibowitz, S., Kennedy, L., \& Lessof, M. (2013). The Tuberculin Reaction in the Pleural Cavity and its Suppression by Antilymphocyte Serum. Br J Exp Pathol, 54(2), 152-62. 


\section{Derrame pleural de origen tuberculoso}

Vol. 3, núm. 3., (2019)

Olga Mariela Herrera Checa; Melina Dennise Medina Gamarra; Marcela Elizabeth Cevallos Sánchez; María Fernanda Sánchez Pilozo

Light, R. (2010). Update on tuberculous pleural effusion. Respirology.

OMS. (10 de Diciembre de 2014). Organización Mundial de la Salud. Informe mundial sobre la tuberculosis 2014,. Ginebra: Organización Mundial de la Salud. Recuperado el 18 de 9 de 2019, de Available online: http://www.who.int/tb/publications/global_report/en/.

Onyenekwu, C. (2013). High pleural fluid adenosine deaminase levels: a valuable tool for rapid diagnosis of pleural TB in a middle-income country with a high TB/HIV burden. . 200225.

Richard, W. (23 de Marzo de 2017). Manual MSD, version para profesionales . Recuperado el 17 de Septiembre de 2019, de https://www.msdmanuals.com/es-ve/professional/trastornospulmonares/trastornos-mediast\%C3\%ADnicos-y-pleurales/derrame-pleural

Seibert, A., Haynes, J., Middleton, \& R. (2012). Derrame pleural tuberculoso. Experiencia de veinte años. .

Sibley, J. (1970). Un estudio de 200 casos de pleuresía tuberculosa con derrame. Amer. Rev. REspir. Dis., 101(1), 838-844.

Smith, I. (2003). Mycobacterium tuberculosis pathogenesis and molecular determinants of virulence. Clin Microbiol , 63-96.

Theron, J. (2010). Direct comparison of the diagnostic yield of ultrasound-assisted Abrams and Tru-Cut needle biopsies for pleural tuberculosis. Thorax, 57-62. .

Tovar, M. (2008). Diagnóstico mejorado de tuberculosis pleural utilizando la técnica de susceptibilidad a fármacos por observación microscópica. 12-34.

Turna, A. (2006). Factores que afectan la morbilidad en el empiema tuberculoso crónico. Thorac Cardiovasc Surg. 